\title{
OSCAR VERGARA: VERDAD O MÉTODO EN LA ÉTICA BIOMÉDICA
}

\section{TRUTH OR METHOD IN BIOMEDICAL ETHICS}

RESUMEN: La moderna hermenéutica filosófica ha realizado una contribución importante al campo de las humanidades. Sin embargo, su aplicación concreta al campo de la ética biomédica ha sido relativamente limitada. Ello, por dos razones. En primer lugar, porque no parece haber un modo de transformar esta contribución en algún tipo de orientaciones concretas para la toma de decisiones. En este trabajo se analizan diversos intentos. En segundo lugar, no siempre ha sido captada adecuadamente la noción de diálogo, la cual resulta capital para que la hermenéutica despliegue todos sus efectos conceptuales. Este trabajo contiene un intento enfocar adecuadamente esta cuestión de acuerdo con el planteamiento que hace Gadamer en Verdad y método.

ABSTRACT: Modern philosophical hermeneutics has made an important contribution to the field of the humanities. Even so, its specific application to the area of biomedical ethics has been relatively limited. This is due to two reasons. In the first place, there seems no way of converting this contribution into some kind of concrete and practical guidance for decision-making. In this work we analyze various attempts. Secondly, the notion of dialogue has not always been properly understood. We can say with no doubt that to grasp the notion in a correct way is indeed of vital importance. Only like this will hermeneutics be able to display all its conceptual implications. This work contains an attempt to focus adequately this issue in line with Gadamer's approach in Truth and Method.

PALABRAS CLAVE: Bioética hermenéutica. Toma de decisiones. Ontología

KEY WORDS: Bioethical Hermeneutics. Decision Making. Ontology

\section{Elementos valorativos en la toma de decisiones}

En el campo del Derecho, la hermenéutica filosófica, sobre todo a partir de Gadamer, ha efectuado una contribución importante para entender cabalmente el proceso de toma de decisiones jurídicas. Se confirma así algo ya puesto de manifiesto por las corrientes antiformalistas de finales del S. XIX y principios del siglo XX; a saber, que la decisión jurídica no es fruto de una mera aplicación mecánica de las normas generales, sino un proceso de concreción o de determinación del Derecho. 
La ética biomédica se distingue del Derecho, entre otras muchas cosas, porque no cuenta con un cuerpo de normas autoritativas que deban ser imperativamente tenidas en cuenta en las decisiones concretas. Esto entraña, por un lado, la dificultad de no contar con criterios válidos a priori; pero, por otro lado, ha permitido salvar más fácilmente el error de pensar que la ética biomédica consista en la aplicación de algún tipo de teoría ética general supuestamente válida.

Otra notable diferencia consiste en la ausencia para la ética biomédica de instituciones autoritativas que adopten decisiones vinculantes. Los procesos para tomar decisiones están escasamente institucionalizados y en ellos participan distintas personas. Por ello, muchos profesionales sanitarios demandan algún tipo de metodología que cuente, si no con autoridad institucional, sí con algún tipo de autoridad moral, sobre todo para abordar los casos moralmente comprometidos.

Esto es difícil de alcanzar, porque en toda decisión hay un componente valorativo insoslayable. La hermenéutica jurídica muestra cómo la decisión está mediatizada por la valoración. El proceso de concreción del Derecho se basa en un silogismo por el cual unos hechos (premisa menor) son subsumidos en el supuesto de hecho de una norma (premisa mayor), de donde se obtiene una conclusión normativa (conclusión). Pero tanto las premisas como la conclusión son valorativas. En primer lugar, la norma hay que seleccionarla e interpretarla. En segundo lugar, los hechos deben ser dispuestos narrativamente de modo que encajen en el supuesto de hecho de la norma. En tercer lugar, la conclusión necesita ser también concretada. Pues bien, todas estas operaciones se efectúan circularmente a través del intérprete, el cual, al ser un sujeto histórico está mediatizado por sus prejuicios ${ }^{1}$.

El bioético no está menos mediatizado por sus prejuicios que el jurista. Aunque recurra a alguna metodología para la toma de decisiones, en el fondo, éstas van a estar basadas en ellos. El principialismo es valorativo porque no determina mecánicamente el resultado. Los principios no se pueden aplicar de un modo rigurosamente lógico. La especificación, que es la operación por la que se obtienen reglas concretas a partir de los principios de autonomía, no maleficencia, beneficencia y justicia, no es una mera concreción interpretativa, sino una decisión normativa ${ }^{2}$.

Por otra parte, el casuismo se basa en la analogía, y ésta consiste precisamente en una valoración acerca de la semejanza o falta de semejanza entre el caso paradigmático y el caso a enjuiciar. Si los casos son parecidos se puede aplicar la misma solución. Pero en la medida en que son parecidos y no idénticos, se puede aplicar también la solución contraria. Es cuestión de valo-

\footnotetext{
${ }^{1}$ Cf. Kaufmann, A., «La espiral hermenéutica», Persona y Derecho 52 (2005), pp. 405-410.

${ }^{2}$ Cf. Vergara, O., «Principlism and Normative Systems», en Serna, P. y Seoane, J.A. (eds.), Bioethical Decision Making and Argumentation, Dordrecht, Springer, 2016, en prensa.
} 
ración. Obviamente, los prejuicios del intérprete ejercerán un peso en favor de una u otra solución ${ }^{3}$.

La hermenéutica es capaz de hacerse cargo de esta realidad, pero no es fácil que pueda ser transformada en un método para la toma de decisiones. En primer lugar, por su carácter filosófico. No es una ética que revista caracteres normativos. En segundo lugar, porque no siempre se enfoca bien la cuestión del diálogo. Ambos problemas se examinan a continuación en sendos epígrafes.

\section{Aproximaciones hermenéuticas a la ética biomédica}

Conviene realizar la siguiente aclaración. La ética biomédica es, ciertamente, ética, pero es también específicamente jurídica. Como enseña Aristóteles, la justicia tiene que ver con el bien ajeno ${ }^{4}$ y en la relación clínica se trata de determinar qué es lo bueno para el paciente. Si el médico va a transfundirle sangre, lo que está en juego es la integridad física y la salud del paciente, no la suya propia.

La hermenéutica gadameriana es útil para las relaciones de justicia porque está dirigida no tanto a la comprensión de uno mismo, cuanto a la comprensión del otro ${ }^{5}$, señaladamente a través de un texto. Precisamente, para K. M. Hunter, el paciente viene a ser semejante a un «texto» que ha de ser comprendido por el médico. A partir de determinados datos -la queja principal del paciente, los otros síntomas, los signos clínicos, los resultados de los análisis, el médico forma una hipótesis que tiene que contrastar nuevamente con los datos, con el fin de afinar o corregir su diagnóstico, en un proceso que por su similitud con el círculo hermenéutico, Hunter denomina "círculo diagnóstico» 6 . En efecto, como señala Gadamer, la salud es algo oculto. Es un estado de medida interna que no es fácil de determinar a través de la simple medición objeti-

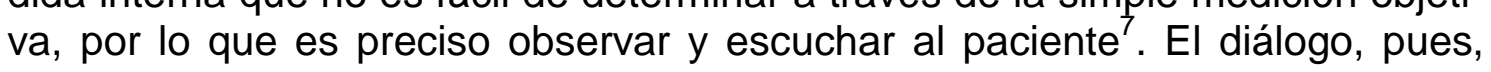
cobra un lugar destacado para un planteamiento hermenéutico.

\section{a) El contextualismo de Thomasma}

Para Thomasma, la razón de ser de la hermenéutica estriba en la necesidad que tienen médico y paciente de comprenderse mutuamente a través de la

\footnotetext{
${ }^{3}$ Cf. Vergara, O., «Casuismo, ética biomédica y Derecho», Persona y Derecho 71 (2014), pp. 323-326.

${ }^{4}$ Cf. ARIStóteles, Ética Nicomáquea, V, 1, 1130a5-7. Se ha consultado la ed. de J. Pallí Bonet, Madrid, Gredos, 2012.

${ }^{5}$ Cf. Gadamer, H.-G., Verdad y método, trad. de A. Agud y R. de Agapito, Salamanca, Sígueme, 2012, p. 394.

${ }^{6}$ Cf. HUNTER, K. M., Doctor's Stories. The Narrative Structure of Medical Knowledge, Princeton, Princeton University Press, 1991, p. 9.

${ }^{7}$ Cf. Gadamer, H.-G., El estado oculto de la salud, trad. de N. Machain, Barcelona, Gedisa, 1996, pp. 115-116.
} 
distancia que inevitablemente los separa. Así, concibe la hermenéutica como un «acto de interpretación a través de los límites»" Para Thomasma, el juicio ético está comprendido en el juicio clínico, por lo que resulta artificial la formulación de una ética aplicada a la medicina. Este rechazo de una ética aplicada es una característica en general de todas las aproximaciones hermenéuticas a la ética clínica.

Thomasma rechaza tanto los modelos decisorios que parten de principios, como los que parten de los casos. En su lugar propone lo que denomina «contextualismo", que, aclara, no es lo mismo que casuismo ${ }^{9}$, y que parte de la idea básica de que el contexto sirve para ajustar los valores en juego a las concretas circunstancias. En su opinión hay una serie de factores de los que depende el peso relativo de los valores y principios en cada caso. Éstos son, principalmente: la especialidad médica implicada; los valores personales del paciente, de su familia o de su grupo social; los valores personales y profesionales del personal sanitario, y el marco institucional en que surge el problema ${ }^{10}$. V. gr., el principio de autonomía tendrá más peso en el contexto de una atención primaria que en el de una atención terciaria, en el cual la autonomía puede verse menguada. Sin embargo, este planteamiento no es excesivamente práctico. Como se puede apreciar, apenas encontramos indicaciones sobre cómo decidir.

b) La teoría interpretativa de Ten Have

El contexto (frente a las teorías aplicadas o los principios abstractos) es también un elemento clave en la «teoría ética interpretativa» de H. Ten Have ${ }^{11}$. Para éste, médico y paciente no son seres al margen de la historia o de la cultura, sino que forman parte de comunidades en que se comparten diversas tradiciones. Su teoría interpretativa pretende, en consecuencia, fundamentarse en los estándares profesionales internos de la práctica asistencial (aunque sin descuidar su conexión con la moralidad externa).

Esta teoría tiene en consideración cuatro parámetros ${ }^{12}$ : a) El punto de partida de la actividad médica es la experiencia moral del paciente. Determinar qué está fallando en éste requiere interpretación. Primero, porque la experiencia de la enfermedad en cada paciente es única e irrepetible. Segundo, debido a que los contextos de interpretación de médico y paciente son distintos y, por

\footnotetext{
${ }^{8}$ ThomasmA, D. C., "Clinical Ethics as Medical Hermeneutics», Theoretical Medicine 15 (1994), p. 95.

${ }^{9}$ Thomasma se separa del casuismo, porque, en su opinión, éste depende de «una teoría unificada de la naturaleza humana y del contexto social» que permita comparar un caso con otro. Este papel lo asumía la teoría del Derecho natural, que hoy, a su juicio, está desacreditada. Cf. ibid., p. 98.

${ }^{10}$ Cf. ibid., p. 100.

${ }^{11}$ Cf. Ten HAVE, H., «The Hyperreality of Clinical Ethics: A Unitary Theory and Hermeneutics», Theoretical Medicine 15 (1994), pp. 118-119.

${ }^{12}$ Cf. ibid., pp. 125-127.
} 
ende, sus precomprensiones. b) Primariamente, la experiencia moral implica sentimientos. Sólo secundariamente pueden éstos ser objeto de reflexión moral. El papel de la ética médica no es tanto explicar y aplicar teorías éticas y principios, sino interpretar y evocar todo lo que abarca la experiencia moral: no sólo los actos y sus efectos, sino también las actitudes y las emociones. c) Hay que considerar, en tercer lugar, que la interpretación de la situación de un paciente no es la actividad individual de un médico, sino que éste realiza su labor guiado por una serie de supuestos culturales sobre la naturaleza del mundo y del cuerpo y la consecuencia de la evolución histórica del conocimiento médico. d) Por último, no hay que descuidar que toda interpretación es tentativa y que siempre es posible más de un significado.

De estos parámetros se desprende que hay que ser consciente de la determinación histórica de toda comprensión. Esto puede ayudar a descartar aquellas soluciones que estén basadas en prejuicios injustificados, pero no determina una solución concreta. Ten Have considera que el diálogo sirve para descubrir las particularidades de nuestros juicios previos y, a través de ello, obtener un mayor grado de comprensión ${ }^{13}$. Esto es correcto. Pero es demasiado genérico para solucionar problemas concretos.

\section{c) La bioética hermenéutica de Leder}

En un sentido similar, Leder opina que las metodologías top-down (v. gr., kantismo, utilitarismo) pueden oscurecer la rica complejidad de los casos. La hermenéutica, en cambio, es capaz de atender a los múltiples contextos, siendo ésta su mayor utilidad ${ }^{14}$. En su opinión, la hermenéutica del s. XX tiende a rechazar la posibilidad de interpretaciones unívocas, admitiendo una indefinida variedad de lecturas. La aproximación hermenéutica consistiría, a su juicio, en escuchar todas las voces de los personajes del drama. En concreto, Leder se alinea con una hermenéutica de la sospecha al modo de Ricoeur, porque, afirma, ni el paciente ni el médico son conscientes de las relaciones subyacentes de poder (v. gr., mercado, consumismo, género, etc. $)^{15}$.

Ver las relaciones sociales en términos de poder es muy discutible. Pero aunque no lo fuera, cabe preguntarse si valen igual todas las mencionadas voces. Tampoco está claro cómo se pasa de la atenta escucha a la práctica. Leder se da cuenta de que no se puede permanecer en una interpretación sin fin, sino que es preciso resolver los casos. Hermenéutica, afirma, no implica relati-

\footnotetext{
${ }^{13}$ Cf. ibid., p. 127.

14 Cf. Leder, D., «Toward a Hermeneutical Bioethics», en DuBose, E.R.; HAmeL, R. \& O'ConnelL, L. J., eds., A Matter of Principle? Ferment in US Bioethics, Valley Forge, Trinity Press International, 1994, p. 251.

${ }^{15}$ Cf. ibid., pp. 252-253. JUNGES, J. R., «Bioética como casuística e como hermenéutica», Revista Brasileira de Bioética 1 (2005), pp. 28-44, propone completar el planteamiento casuístico con una hermenéutica de la sospecha que interprete los presupuestos éticos, antropológicos y socio-culturales que determinan la manera como las realidades relacionadas con la vida y la salud son comprendidas en la sociedad y la cultura actuales (p. 41).
} 
vismo. A su juicio, hay una base que permite superar la subjetividad, que es la tradición compartida ${ }^{16}$. Asimismo, opina, el diálogo permite tomar conciencia de los inevitables prejuicios que acompañan a la comprensión. Ante una sociedad, como la occidental, dividida, el hermeneuta debe contribuir a articular las perspectivas de los participantes, trayendo al debate los diferentes contextos. Su papel, precisa, no es el de hombre-respuesta, sino el de interlocutor socrático, a través de la invitación al diálogo ${ }^{17}$.

Todo esto es interesante, aunque insuficiente para determinar la interpretación correcta. La hermenéutica no aporta un método, sino una toma de conciencia acerca de nuestra mediatizada capacidad para alcanzar la verdad. Pero cabe preguntarse si no será preciso presuponer dicha verdad, porque, en otro caso, no está claro qué sentido tiene dialogar.

\section{d) La hermenéutica platónica de Lingiardi y Griego}

De un modo todavía más indeterminado, Lingiardi y Grieco proponen que el médico se convierta en filósofo ${ }^{18}$. Afirman que es preciso que entre médico y paciente se forme un verdadero diálogo. El paciente no puede estar en una posición de mera pasividad. Pero tampoco se trata sólo de escuchar al paciente. La tarea del filósofo consiste en preguntar. Para estos autores es importante advertir que el médico comparte con el paciente un cuerpo mortal, es también vulnerable. Un médico «con heridas», opinan, puede activar la capacidad curativa del paciente ${ }^{19}$. Esto va más allá de la mera empatía y se fundamenta en la idea platónica de médico. Éste no es alguien que dispense desde arriba, sino alguien que simplemente inicia en otro el proceso de curación ${ }^{20}$.

A la luz de lo anterior podemos afirmar con Cadorè que la aproximación hermenéutica a la ética clínica «es diferente de un método para resolver problemas" ${ }^{21}$. Es significativo que Gadamer en su El estado oculto de la salud, se abstenga totalmente de formular algo así como un método para la ética biomédica $^{22}$.

\footnotetext{
${ }^{16}$ Como señala Lolas, en el juicio moral, la cultura, la tradición y la costumbre hablan antes que la razón individual. El proceso de deliberación moral lo que hace es afinar o modificar esas primeras impresiones. $Y$ cita a Ortega: «En las creencias se está y las ideas se tienen». Cf. LOLAS, F., "De la praxis al texto, del texto a la praxis: La bioética como hermenéutica», Acta biológica 3 (2009), p. 4.

${ }^{17}$ Cf. LeDER, D., art. cit., p. 255.

${ }^{18}$ Cf. LINGIARDI, V, GRIECO, A., «Hermeneutics and the Philosophy of Medicine: Hans-Georg Gadamer's Platonic Metaphor», Theoretical Medicine and Bioethics 20 (1999), p. 419.

${ }^{19}$ Cf. ibid., pp. 419-420.

${ }^{20}$ Cf. ibid., p. 413.

${ }^{21}$ CAdorè, B., «A Hermeneutical Approach to Clinical Bioethics", en VIAFORA, C. Clinical Bioethics: A Search for the Foundations, Dordrecht, Springer, 2005, p. 56.

${ }^{22}$ GadAmer, H.-G., El estado oculto de la salud, cit..., passim.
} 


\section{El diálogo y la cuestión ontológica}

Afirma Gadamer: «Lo que no logra la herramienta del método tiene que conseguirlo, y puede realmente hacerlo, una disciplina del preguntar y el investigar que garantice la verdad ${ }^{23}$.

Todos los planteamientos hermenéuticos anteriores hacen hincapié en la necesidad de que médico y paciente se escuchen mutuamente. Pero se trata de una escucha activa que tiene lugar a través del diálogo. Éste permite el mutuo cuestionamiento de los prejuicios que van constitutivamente unidos a todo acto de comprensión y que en algunos casos pueden sesgar dicha comprensión. Esta característica del diálogo no es una apertura psicológica al otro, entendida como mera empatía, un ponerse en el lugar del otro, sino que es una apertura de índole ontológica. Tiene parte de razón Svenaeus cuando afirma que: «La verdad en la filosofía de Gadamer [...] ha de ser primariamente entendida como apertura al otro y a su mundo y no sólo a mi propio mundo ${ }^{24}$.

Pero esta afirmación no es suficiente. Porque radicalmente no se trata de ni de mi verdad, ni de su verdad, sino de la verdad a la que, a pesar de las inevitables condiciones subjetivas de la comprensión, tanto él como yo, podemos de algún modo acceder. No se trata ni de que yo me imponga ni de que se imponga el otro, como en una relación de poder. La relación entre médico y paciente, a diferencia de lo que plantea Leder, no es primariamente una relación de poder entre los sujetos de una relación. De lo que se trata es de que sea la cosa misma, su verdad, la que se imponga.

Por ello Gadamer afirma: «No cabe duda de que para el comprender lo decisivo sigue siendo entender la cosa, adquirir una percepción objetiva; no se trata de un procedimiento histórico ni de un procedimiento psicológicogenético ${ }^{25}$. $Y$, como señala en otro contexto, hay algo que tienen en común la comprensión de un texto y el ponerse de acuerdo en una conversación: que toda comprensión y todo acuerdo tienen presente alguna cosa que uno tiene ante sí; en ambos casos, de lo que se trata es de dejar hablar a la cosa mis$m a^{26}$. Si el paciente no tiene un problema de salud -en el sentido más amplio del término-, el diálogo entre médico y paciente (como tales) carece de sentido.

Es un error pensar que de lo que se trata con la comprensión es únicamente de desvelar el sentido subjetivo de la intención del autor del texto. Cuando Gadamer recurre al diálogo y al método socrático de la pregunta como modo de encaminarse hacia la verdad, lo hace sobre la base de permitir así

\footnotetext{
${ }^{23}$ GAdAMER, H.-G., Verdad y método, cit., p. 585.

${ }^{24}$ SVEnAEUS, F., «Hermeneutics of Medicine in the Wake of Gadamer: The Issue of Phronesis», Theoretical Medicine 24 (2003), p. 414

${ }^{25}$ GadAmer, H.-G., Verdad y método, cit., p. 236.

${ }^{26}$ Cf. ibid., p. 457.
} 
que las cosas mismas afloren y se hagan valer, frente a las opiniones y prejuicios que le dominan a uno ${ }^{27}$.

No es, pues, correcto reducir lo hermenéutico a un diálogo de perspectivas, en que médico, paciente, miembros del comité, etc. efectúan una aproximación de posturas al acercarse recíprocamente a otros horizontes de comprensión ${ }^{28}$. Es un hecho que esto se produce. La cuestión es por qué se produce. En sentido radical, ¿por qué aproximar lo que está distante? Si todas las perspectivas, médico, paciente, etc. son válidas, lo congruente es intentar imponerlas en una lucha de poder. Si esto no es de hecho así, ello no es porque, al modo hobbesiano, así se sustituya la discordia por la concordia, la guerra por la paz. No es una cuestión psicológica, sino ontológica. El diálogo, si hay una actitud adecuada, permite descubrir y superar aquellas posiciones que no sintonizan con la realidad. Pero esto presupone que, por muchas facetas que presente, existe algún tipo de acceso, si bien parcial, a la verdad objetiva.

Es verdad, como afirma Svenaeus, que Gadamer no considera que el fin de la comprensión hermenéutica sean verdades intemporales que puedan ser alcanzadas a través de algún método universal e intemporal. La verdad, señala acertadamente Svenaeus, es siempre concreta y depende del encuentro de dos horizontes concretos de comprensión, un encuentro que se dirige a la realización de algún fin (goal) ${ }^{29}$. Este fin es sanar (healing). Comoquiera que «sanar es una cosa buena (good thing)», un fin moralmente valioso, la hermenéutica de la medicina presenta una "estructura normativa» ${ }^{30}$.

Este planteamiento es claramente aristotélico. En efecto, para Aristóteles la phrónesis o prudencia, que es la virtud encargada de la decisión, es normati$\mathrm{va}^{31}$ por la razón que se acaba de señalar. Pero esta virtud, aunque de suyo es de carácter intelectual, no es practicable sino sobre la base de la virtud moral, como reconoce Svenaeus. El propio diálogo requiere una voluntad que esté abierta a la realidad de las cosas, a aceptar el error propio y la verdad ajena. Sin esta base se convierte en un arma meramente retórica.

A pesar de ello, y reconociendo que la ética de la virtud es una de las posibilidades de desarrollar una ética que gire alrededor de la phrónesis, Svenaeus apuesta por la fenomenología hermenéutica ${ }^{32}$. Y aunque no tendría por qué ser así, Svaneaus acaba regresando al punto de partida, reduciendo la comprensión a un fenómeno psicológico en el que el médico se abre al horizonte del paciente, a sus preferencias y a sus ideas, para lo que el diálogo entre

\footnotetext{
${ }^{27}$ Cf. ibid., p. 556.

${ }^{28}$ Cf. Svenaeus, F., «Hermeneutics of Clinical Practice: The Question of Textuality», Theoretical Medicine and Bioethics 21 (2000), pp. 181-183.

${ }^{29}$ Cf. SvenAeUS, F., «Hermeneutics of Medicine in the Wake of Gadamer», cit., p. 417.

${ }^{30}$ Ibidem.

${ }^{31}$ Cf. ARIStÓteles, Ética a Nicómaco, cit., VI, 10, 1143a8-9.

${ }^{32}$ Cf. Svenaeus, F., «Hermeneutics of Medicine in the Wake of Gadamer», cit., p. 425.
} 
médico y paciente resulta fundamenta ${ }^{33}$. En suma, para Svenaeus, el «buen hermeneuta de la medicina» es phronético en el sentido de que es dialógicamente competente (dialogically skilful) ${ }^{34}$.

Pero esto es una reducción del planteamiento aristotélico. Ser phronético no equivale a ser dialógicamente competente. Ser dialógicamente competente puede ser un medio para ser phronético. Pero ser phronético en Aristóteles consiste en ser capaz de encontrar los medios que se dirigen a vivir bien en genera $\left.\right|^{35}$. Pero vivir bien en general no está a disposición de la voluntad, sino que es la voluntad la que debe estar dispuesta a vivir bien en general.

No es exacta la afirmación de Svenaeus según la cual la medicina es una actividad phronética ${ }^{36}$. La medicina es más bien una actividad técnica que participa en cierta medida de la phrónesis. Es técnica porque se dirige a un bien concreto, la salud, no a vivir bien en general. Por otra parte la prudencia, como enseña Aristóteles, delibera sobre lo que es bueno y conveniente para uno mismo $^{37}$. Sin embargo, el médico delibera sobre lo que es bueno para el paciente $^{38}$. Éste sí que delibera sobre lo que es bueno para sí mismo, por lo que su decisión es (o debería ser) primariamente phronética. Para el médico, lo es derivativamente, en la medida en que sea capaz de ponerse en su lugar. Primariamente, lo es en la medida en que su ejercicio profesional forma parte, obviamente, de su proyecto de vida.

Puede suceder que ambos proyectos de vida desencadenen decisiones contrapuestas. V. gr., el médico indica una transfusión de sangre a un testigo de Jehová, que la rechaza. Pero hay que tener presente que más allá de los bienes particulares de cada sujeto está el bien común. La política supera a la ética, pero abarcándola y haciéndola suya ${ }^{39}$. Ambos sujetos deben interpretar no sólo el contexto en que el otro toma una decisión, sino también el contexto del que ambos forman parte. La profesión médica se dirige al bien común y el bien que trata de hacer valer el paciente también lo es para el médico y la sociedad en su conjunto. No hay una lucha de intereses. No se trata de que el médico, como individuo, ceda o que se pase por encima de su proyecto de vida buena. Recomendar un tratamiento alternativo, si ello es posible, forma parte también de un proyecto de vida buena.

\footnotetext{
${ }^{33}$ Cf. ibid., p. 426; SVenaeUS, F., «Hermeneutics of Clinical Practice», cit., p. 178.

${ }^{34}$ Cf. Svenaeus, F., «Hermeneutics of Medicine in the Wake of Gadamer», cit., p. 425.

${ }^{35}$ ARISTÓTELES, Ética a Nicómaco, cit., VI, 5, 1140a25-27.

${ }^{36}$ Para Svenaeus, la medicina está más próxima a la phrónesis que a la techne. Cf. ibid., p. 421.

${ }^{37}$ Ibidem.

${ }^{38}$ De modo análogo, en el mundo del Derecho, el justo es el que quiere dar a cada uno lo suyo. El jurista es el que sabe qué es lo suyo de cada uno. En cuanto a la medicina, el paciente es el que quiere la salud; el médico, el que sabe cómo la puede recuperar.
}

${ }^{39}$ ARISTÓteles, Ética a Nicómaco, cit., I, 2. 


\section{Conclusiones}

En todo proceso decisorio se presenta un insoslayable elemento valorativo, del que no se hacen cargo las metodologías bioéticas al uso, el cual ha sido, en cambio, bien teorizado por la moderna hermenéutica filosófica. Algunos planteamientos bioéticos han adoptado una orientación hermenéutica para abordar la cuestión de la toma de decisiones. Sin embargo, se muestran insuficientes, debido a que, como enseña Aristóteles, la sabiduría (la hermenéutica es un conocimiento teórico) no hace a las personas necesariamente prudentes. Por otra parte, los mencionados planteamientos, aunque giran en torno a la noción capital de diálogo, no le hacen justicia y no son capaces de captar su sentido profundo, que no es de índole psicológica, sino ontológica. Es, en definitiva, más una cuestión de verdad que de método.

\section{Bibliografía citada}

Aristóteles. Ética a Nicómaco: VI, 10, 1143a8-9. Pallí, J., ed. Madrid: Gredos; 2010.

Cadorè, B. "A Hermeneutical Approach to Clinical Bioethics". En: Viafora, C. Clinical Bioethics: A Search for the Foundations. Dordrecht: Springer; 2005: 53-59.

Gadamer, H.-G. El estado oculto de la salud. Trad. de N. Machain. Barcelona: Gedisa; 1996.

Gadamer, H.-G. Verdad y método. Trad. de A. Agud y R. de Agapito. Salamanca: Sígueme; 2012.

Hunter, K. M. Doctor's Stories. The Narrative Structure of Medical Knowledge. Princeton: Princeton University Press; 1991.

Junges, J. R., "Bioética como casuística e como hermenéutica". Revista Brasileira de Bioética 2005; 1: 28-44.

Kaufmann, A., «La espiral hermenéutica», Persona y Derecho 52 (2005), pp. 405-410.

Leder, D. "Toward a Hermeneutical Bioethics". En: DuBose, E.R.; Hamel, R. \& O'Connell, L. J., eds. A Matter of Principle? Ferment in US Bioethics. Valley Forge: Trinity Press International; 1994: 240-259.

Lingiardi, V, Grieco, A., "Hermeneutics and the Philosophy of Medicine: HansGeorg Gadamer's Platonic Metaphor". Theoretical Medicine and Bioethics 1999; 20: 413-422.

Lolas, F. "De la praxis al texto, del texto a la praxis: La bioética como hermenéutica". Acta biológica 2009; 3: 29-41.

Svenaeus, F. "Hermeneutics of Clinical Practice: The Question of Textuality". Theoretical Medicine and Bioethics 2000; 21: 171-189.

Svenaeus, F. "Hermeneutics of Medicine in the Wake of Gadamer: The Issue of Phronesis". Theoretical Medicine 2003; 24: 407-431.

Ten Have, H. "The Hyperreality of Clinical Ethics: A Unitary Theory and Hermeneutics". Theoretical Medicine 1994; 15: 113-131. 
Thomasma, "D. C. Clinical Ethics as Medical Hermeneutics". Theoretical Medicine 1994; 15: 93-111.

Vergara, O., «Casuismo, ética biomédica y Derecho», Persona y Derecho 71 (2014), pp. 307-333.

Vergara, O., «Principlism and Normative Systems», en SERNA, P. y SEOANE, J.A. (eds.), Bioethical Decision Making and Argumentation, Dordrecht, Springer, 2016, pp. 57-71. 